Schweiz. Gastroenterol. 2020 1 1:121-129 https://doi.org/10.1007/s43472-020-00021-z Online publiziert: 12 . Oktober 2020 (c) Der/die Autor(en) 2020

\section{B. Morell · F. O. The $\cdot$ C. Gubler ${ }^{1}$}

'Klinik für Gastroenterologie und Hepatologie, UniversitätsSpital Zürich, Zürich, Schweiz

\title{
Die flexible Endosonographie in der Gastroenterologie: die Entwicklung von der Diagnostik zur Therapie
}

Endosonographie in der Gastroenterologie bedeutet, dass ein Endoskop am distalen Ende mit einem kleinen Ultraschallkopf bestückt ist. Je nach Einsatzgebiet sind diese Endoskope starr oder flexibel konfiguriert. Die starren Endoskope besitzen keine Optik und sind naturgemäss nur anal mit geringer Einführtiefe zu verwenden. Diese erlauben eine Beurteilung des Analkanals und des distalen Rektums. Für den Einsatz von oral mit Beurteilung des Ösophagus bis und einschließlich des Duodenums oder bei Verwendung oralseits des Rektums wird ein flexibles Endoskop mit Optik als Träger für den Ultraschallkopf benötigt. Da der Schallkopf am distalen Ende sitzt, ist die Optik selbst lateral positioniert, sodass man von einer Seitoptik spricht, die eine entsprechende Übung zur Bedienung voraussetzt. Die flexible Endosonographie (EUS) kann mit einem 360-GradSchallkopf durchgeführt werden, was eine Beurteilung der Wand von Ösophagus bis zum Analkanal als Schnittbild senkrecht zur Achse des Gastrointestinaltrakts erlaubt. Diese Technik wurde 1980 eingeführt und ist rein diagnostischer Natur. Sind die Piezokristalle nur streifenförmig auf einer konvexen Oberfläche aufgereiht, wird mit geringer Ankopplungsfläche ein relativ grosser Ausschnitt betrachtet werden können; allerdings nur in einer Ebene. Diese Transducer nennt man Konvexscanner und sie werden beim transabdominalen und endoskopischen Ultraschall eingesetzt. Verwendet man einen solchen Konvexscanner als Schallkopf auf dem Endoskop, können mit Nadeln über einen zusätz- lichen, proximal gelegenen Arbeitskanal Fokalbefunde unter EUS-Sicht diagnostisch angegangen werden. Mithilfe eines seilzugkontrollierten Hebels am Ende des Geräts (Albarrán-Hebel) sind die Nadeln in dieser einen Ebene exakt steuerbar (• Abb. 1).

Diese EUS mit Feinnadelpunktion wurde 1992 erstmalig beschrieben und ermöglicht einen wenig invasiven diagnostischen Zugang bei vielen Läsionen mediastinal, retroperitoneal oder infraperitoneal. Haupteinsatzgebiet ist die Gewebsdiagnostik bei Tumoren, Lymphknotenveränderungen, der linken Nebenniere oder bei perigastralen Läsionen. Letztendlich muss der Befund innerhalb von $2-6 \mathrm{~cm}$ Reichweite ab Wand im Gastrointestinaltrakt liegen und die Eindringtiefe des Endoskops erreicht diese Lokalisation auch. So erreicht das EUS-Gerät von oral Läsionen bis und mit Pars III duodeni und von anal kann bis und mit Colon sigmoideum sinnvoll gearbeitet werden.

Je nach Wahl und Technik der Nadel und des Untersuchers werden Feinnadelaspirationszytologien (FNA) oder Feinnadelbiopsien (FNB) gewonnen. Die EUS-Bild-Generierung erlaubt zudem semiquantitativ die Bestimmung der Elastizität. Diese EUS-Elastographie gibt hart und weich in einer fliessenden Farbskala an, typischerweise sind „harte“ Läsionen blau und oft maligner Natur (•Abb. 2). Die per Knopfdruck zuschaltbare Technik erlaubt die Beurteilung von schwierig abgrenzbaren Raumforderungen und kann beim Aufsuchen von Fokalbefunden oder patho- logischen Lymphknoten sehr hilfreich sein. Die zusätzliche Applikation von parenteralem Kontrastmittel als Bolus verstärkt die Sensitivität und Spezifität der EUS und wird „contrast-enhanced EUS“ (CE-EUS) genannt. Die RealtimeBeurteilung kann sehr gut zwischen perfundierten und nichtperfundierten Arealen unterscheiden. Dies kann bei der Unterscheidung von Schlick gegenüber echten Knoten in Pankreaszysten hochrelevant sein. Zudem kann das Kontrastmittelverhalten Hinweise auf die Dignität einer Läsion geben. Bleibt nach der früharteriellen Phase das Kontrastmittel nicht in der Läsion, spricht man von einem „wash-out", was ebenfalls für eine maligne Läsion spricht.

Letztendlich ist seit kurzer Zeit die EUS-Nadel-basierte Endomikroskopie genannte konfokale Laserendomikroskopie im klinischen Alltag verfügbar. Nötig ist hierfür die parenterale Applikation von Fluoreszin kurz vor der Punktion/Inspektion von Läsionen.

Für die Durchführung der konfokalen Laserendomikroskopie wird eine Lasersonde durch eine 19Gauge-Punktionsnadel in eine zystische Pankreasläsion eingeführt und die Zystenwand mittels eines Lasers mit geringer Energie „,illuminiert", sodass reflektierte elektromagnetische Wellen detektiert werden können. Ein Prozessor generiert eine hochauflösende Darstellung der mukosalen Histologie. Anhand dieser „optischen Biopsie“ kann das Gewebe weiter charakterisiert werden, d. h., es kann z. B. zwischen einer IPMN (Intraductalen papillären muzinösen Neoplasie) und einem makrozys- 


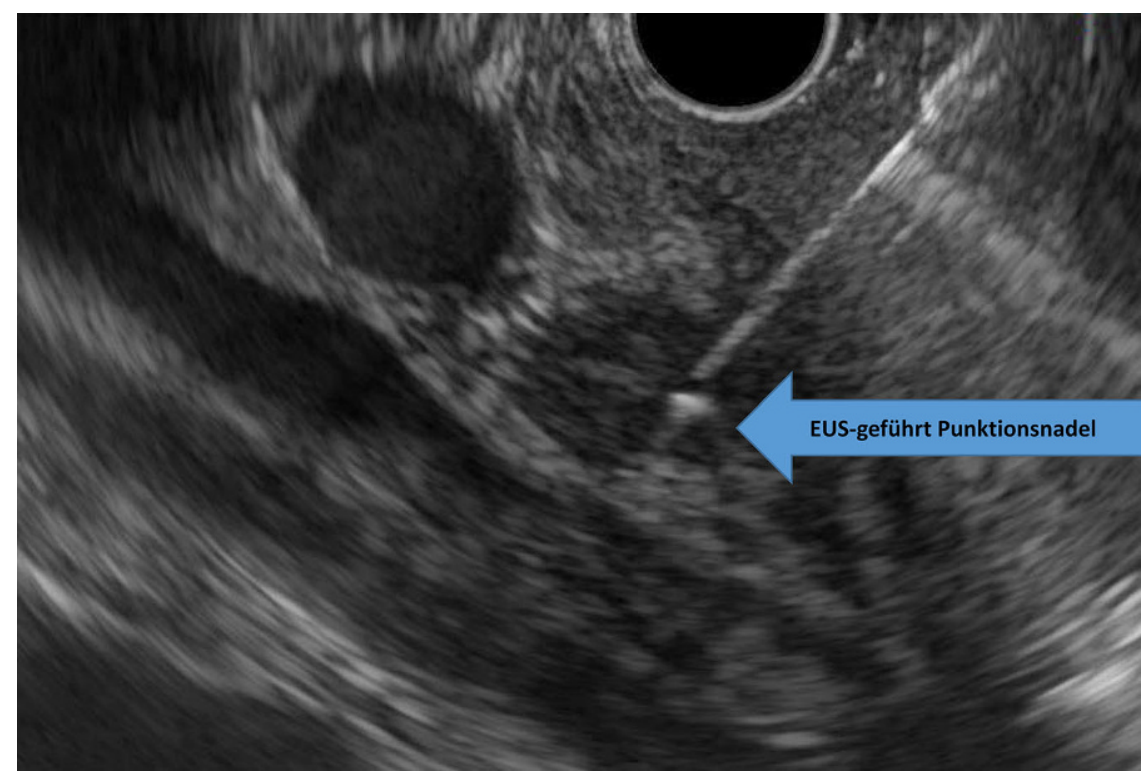

Abb. 1 ॥ Spitze der Punktionsnadel im Zentrum des Lymphknotens. EUS Endosonographie

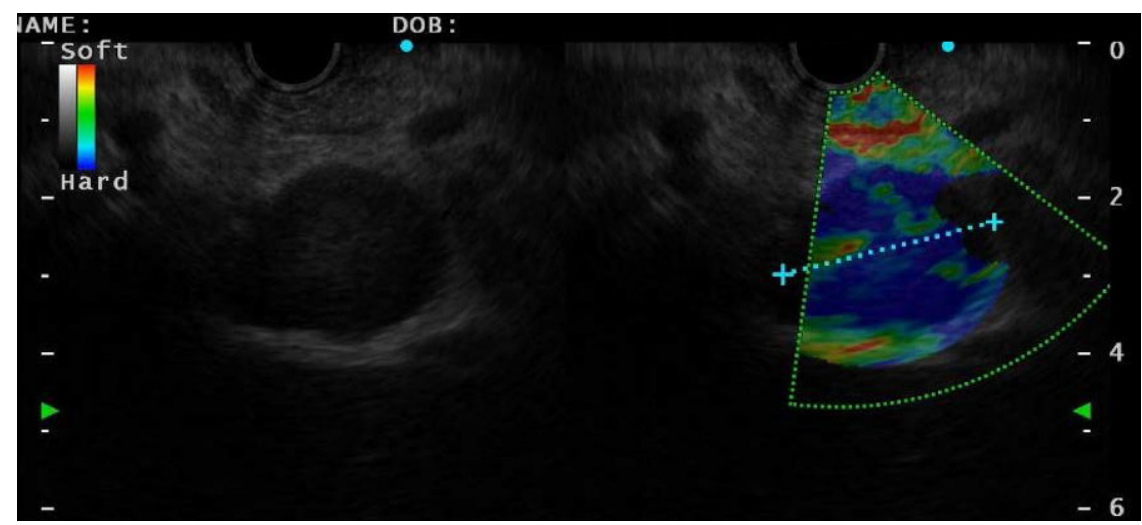

Abb. 2 ॥ „Harte“ Elastographie eines entrundeten Lymphknotens = malignomverdächtig

tischen serösen Zystadenom (• Abb. 3) unterschieden werden [1].

In den letzten Jahren hat sich jedoch die EUS schnell von der reinen diagnostischen Untersuchung zu einem wenig invasiven therapeutischen Eingriff weiterentwickelt. Diese sog. interventionelle EUS macht den verwandten Disziplinen der Radiologie, Chirurgie wie auch etablierten gastroenterologischen Eingriffen wie z.B. der ERCP (endoskopische retrograde Cholangiopankreatikographie) zunehmend ernsthafte Konkurrenz.

Prinzipiell können wie folgt interventionelle EUS-Eingriffe erfolgen:

- Über die EUS-gesteuerte Nadel können Substanzen appliziert werden oder z. B. Radiofrequenzkatheter eingeschoben werden zur Destruktion von fokalen kleinen Läsionen.

\section{Nadelbasierte Interventionen}

Eine seit Jahren etablierte und wenig bekannte Methode ist die EUS-geführte Truncus-coeliacus-Blockade zur anhaltenden Therapie bei Patienten mit chronischer therapierefraktärer Schmerzsymptomatik. Hier handelt es sich einerseits um Patienten mit chronischer Pankreatitis wie auch um Patienten mit einem Pankreaskarzinom in palliativer Konstellation. Dieser Block stellt eine valable Alternative zur chronischen problematischen Opiattherapie oder der perkutanen Trunkusblockade dar. Mithilfe der linearen EUS-Sonde kann der Truncus coeliacus transgastral in den meisten Fällen sehr gut identifiziert, oft können auch die Ganglien selbst abgegrenzt werden. Mit einer Hohlnadel wird dann probatorisch ein langwirksames Lokalanästhetikum unter Ultraschallkontrolle in die Ganglien oder in diese Region appliziert. Zeigt dieser Block anhaltend eine gute klinische Wirkung, kann in einem 2. Schritt eine Neurolyse durchgeführt werden. Hier wird mit der gleichen Methode $96 \%$ iger Alkohol zur Destruktion der Ganglien eingespritzt. Mit dieser Methode ist in über $50 \%$ eine gute Linderung der Schmerzen und selten eine vollständige Schmerzfreiheit zu erwarten. Der Effekt kann in 10-20\% sogar für Monate anhalten [2].

Zur stereotaktischen Bestrahlung benötigen die Kollegen der Radioonkologie röntgendichte Marker. Diese sog. Fiducials können mithilfe der EUS auf den Millimeter genau im Gewebe platziert werden. Mithilfe einer vorgeladenen EUSNadel ist es sodann möglich, z. B. Pankreastumoren gut in 2 Ebenen zu markieren und somit radiologisch zu lokalisieren.

Bei der portalen Hypertension jedweder Ätiologie treten Varizen nicht nur ösophageal auf, sondern können weniger häufig auch als Magenvarizen symptomatisch werden. Diese Magenvarizen haben eine höhere Mortalität und sind aufgrund ihrer Topographie und Gefässanatomie schwieriger zu behandeln. Eine einfache Gummibandligatur ist nicht zielführend. Relevant für die Therapieplanung sind zudem nur die transmuralen Gefässe, die ins Lumen bluten können. Die in der Schichtbildgebung oft imposanten 
Schweiz. Gastroenterol. 2020 1:121-129 https://doi.org/10.1007/s43472-020-00021-z

(c) Der/die Autor(en) 2020

\section{B. Morell · F. O. The · C. Gubler \\ Die flexible Endosonographie in der Gastroenterologie: die Entwicklung von der Diagnostik zur Therapie}

\section{Zusammenfassung}

Die Endosonographie (EUS) in der Gastroenterologie hat sich von der rein diagnostischen Modalität zur vielversprechenden therapeutischen Disziplin gewandelt. Erster Schritt in diese Richtung war das EUS-gesteuerte Entnehmen von Gewebsproben an schwierig zugänglichen Lokalisationen; hier ist die Feinnadelpunktion von Pankreasläsionen an vorderster Stelle zu nennen. Mit dem EUS-gesteuertem Punktieren werden aber auch Gefässe erreicht, sodass Varizen gezielt verödet werden können. Kollektionen nach einer Pankreatitis werden ebenfalls minimalinvasiv angegangen und werden so nach luminal drainiert, statt eine chirurgische Intervention nötig zu machen. Durch das Einbringen von Führungsdrähten über die Hohlnadel sind zudem verschiedene Zugänge der Gallenwege intra- und extrahepatisch möglich, sodass interne Drainagen bei frustraner ERCP (endoskopische retrograde Cholangiopankreatikographie) möglich werden. Durch das Einbringen von speziell konfigurierten beschichteten Stents wiederum werden mit der interventionellen EUS neue Zugänge in Hohlorgane minimalinvasiv als Alternative zu chirurgischen Eingriffen geschaffen. Als Beispiel ist hier die endoskopische Gastroenterostomie bei maligner Magenausgangsstenose zu nennen.

Schlüsselwörter

Interventionelle Endosonographie · Feinnadelpunktion · Selbstexpandierender Metallstent (SEMS) · Lumen-apposing-metal-Stents (LAMS) · Extraanatomische Zugänge

\section{L'échographie endoscopique flexible en gastro-entérologie: son évolution du diagnostic au traitement}

\section{Résumé}

L'échographie endoscopique (EUS) en gastroentérologie, autrefois une modalité purement diagnostique, est devenue une discipline thérapeutique prometteuse. Le premier pas dans cette direction a été le prélèvement d'échantillons tissulaires guidé par EUS dans des localisations difficiles d'accès; mentionnons ici en premier lieu la biopsie à l'aiguille fine de lésions pancréatiques. Les ponctions guidées par EUS permettent aussi d'atteindre des vaisseaux sanguins, et ainsi de traiter les varices par une sclérothérapie ciblée.
Les collections à la suite d'une pancréatite sont également réalisées de façon miniinvasive avec drainage en direction luminale, ce qui permet d'éviter la nécessité d'une intervention chirurgicale. L'insertion d'un filguide glissé dans l'aiguille creuse permet en outre différents accès aux voies biliaires intrahépatiques et extrahépatiques, offrant la possibilité de drainages internes lors d'échec de la cholangiographie rétrograde pancréatique endoscopique. Dans le cadre de l'EUS interventionnelle, la mise en place de stents enrobés spécialement configurés crée - par un procédé mini-invasif au lieu de chirurgical - de nouveaux accès à des organes creux. On peut mentionner ici l'exemple de la gastro-entérostomie endoscopique lors de sténose maligne à la sortie de l'estomac.

Mots clés

Échographie endoscopique $\cdot$ Ponction à l'aiguille fine - Stent métallique autoexpansif (SEMS) · Lumen-Apposing Metal Stents (LAMS) · Accès extra-anatomiques

\section{La flessibilità dell'endosonografia in gastroenterologia: lo sviluppo da diagnostica a terapia}

\section{Riassunto}

In gastroenterologia, l'endosonografia (EUS/endoscopic ultrasound) è passata da modalità puramente diagnostica a disciplina terapeutica molto promettente. II primo passo in questa direzione è stata la raccolta, sotto guida EUS, di campioni di tessuto in punti difficili da raggiungere; a questo riguardo va menzionata innanzitutto la puntura con ago fine delle lesioni pancreatiche. Tuttavia, l'esecuzione di punture sotto guida EUS consente anche di raggiungere i vasi sanguigni in modo da poter sclerosare le varici in modo mirato. Accumuli successivi a una pancreatite vengono affrontati in modo minimamente invasivo e vengono quindi drenati in direzione luminale invece di ricorrere a un intervento chirurgico.

Inserendo fili guida attraverso l'ago cavo, è possibile accedere in vari modi ai dotti biliari intra ed extraepatici, in modo da rendere possibili drenaggi interni in caso di insuccesso dell'ERCP [endoscopic retrograde cholangiopancreatography]. Mediante l'inserimento di stent rivestiti appositamente configurati,
l'EUS interventistica crea nuovi accessi agli organi cavi con una procedura minimamente invasiva alternativa all'intervento chirurgico. Un esempio da indicare al riguardo è la gastroenterostomia endoscopica per la stenosi maligna del piloro.

\section{Parole chiave}

Endosonografia interventistica $\cdot$ Puntura con ago fine - Stent metallico autoespandibile (SEMS) - Lumen-Apposing Metal Stents (LAMS) · Accessi extra-anatomici
Kollateralen ohne Penetration der Wand sind für die Patienten nicht bedrohlich. Mithilfe der EUS kann man diese intramuralen penetrierenden Varizen gezielt und kontrolliert mit Klebstoff oder Coils ausfüllen und so die Blutung auch bei schlechter endoskopischer Sicht stillen. Als Komplikationen sind Infektionen, selbstlimitierte Blutungen und Embolisationen von Fremdkörpermaterial zu nennen, die bei vorhandener Expertise mit etwa $5 \%$ zu beziffern sind. Die techni- sche Erfolgsrate mit klinischem Ansprechen ist hoch und mit $>95 \%$ zu erwarten [3].

Als neueste Errungenschaft sind Radiofrequenzkatheter von 2 kommerziellen Anbietern erhältlich, die durch die 


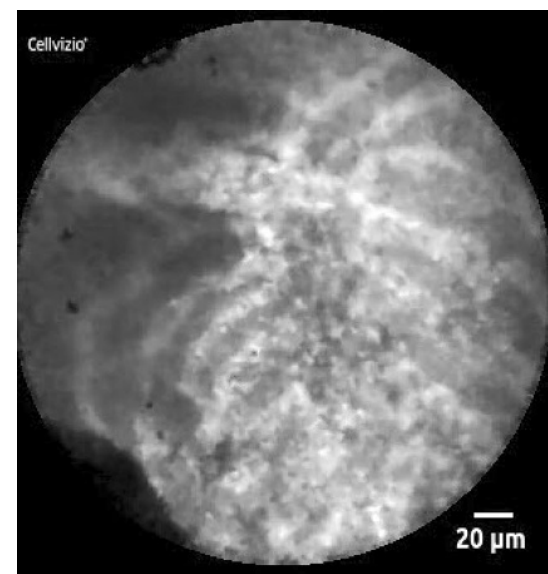

Abb. $3 \Delta$ Endomikroskopie eines serösen Zystadenoms des Pankreas (,superficial vascular network")

EUS-Hohlnadel anwendbar sind. Mithilfe dieser Katheter sind somit Tumoren oder Lymphknoten durch Hitze destruierbar. Die maximal behandelbare Grösse ist durch das Hitzefeld der Nadel definiert und misst $2-3 \mathrm{~cm}$ im Durchmesser. Tumoren müssen somit vom GI-Trakt mit der EUS vom Lumen her erreichbar sein. Idealerweise können so pankreatische Tumoren wie z. B. Insulinome wenig invasiv behandelt werden, statt einen chirurgischen Eingriff nötig zu machen. Es ist auch als weitere Indikation möglich, dysplastische Knoten in pankreatischen zystischen Tumoren definitiv zu abladieren, ohne eine chirurgische Resektion nötig zu machen. Dies kann bei älteren polymorbiden Patienten eine gute Option mit tiefer Morbidität darstellen. Beim jetzigen Stand des Wissens ist bei kleinen pankreatischen neuroendokrinen Tumoren von einer Ablationsrate von $85 \%$ auszugehen, dies bei einem Follow-Up von durchschnittlich 12 Monaten [4].

\section{2.a Zugang zu Kollektionen}

Eine akute schwere Pankreatitis kann zu peripankreatischen Flüssigkeitskollektionen retroperitoneal/abdominal oder zu pankreatischen Nekrosen führen. Diese Kollektionen wiederum reifen innert Wochen $\mathrm{zu}$ definierten Arealen meist perigastral oder periduodenal. Seltener sind die Kollektionen entfernter von Magen oder Duodenum und lokalisieren sich in die parakolischen Rinnen oder
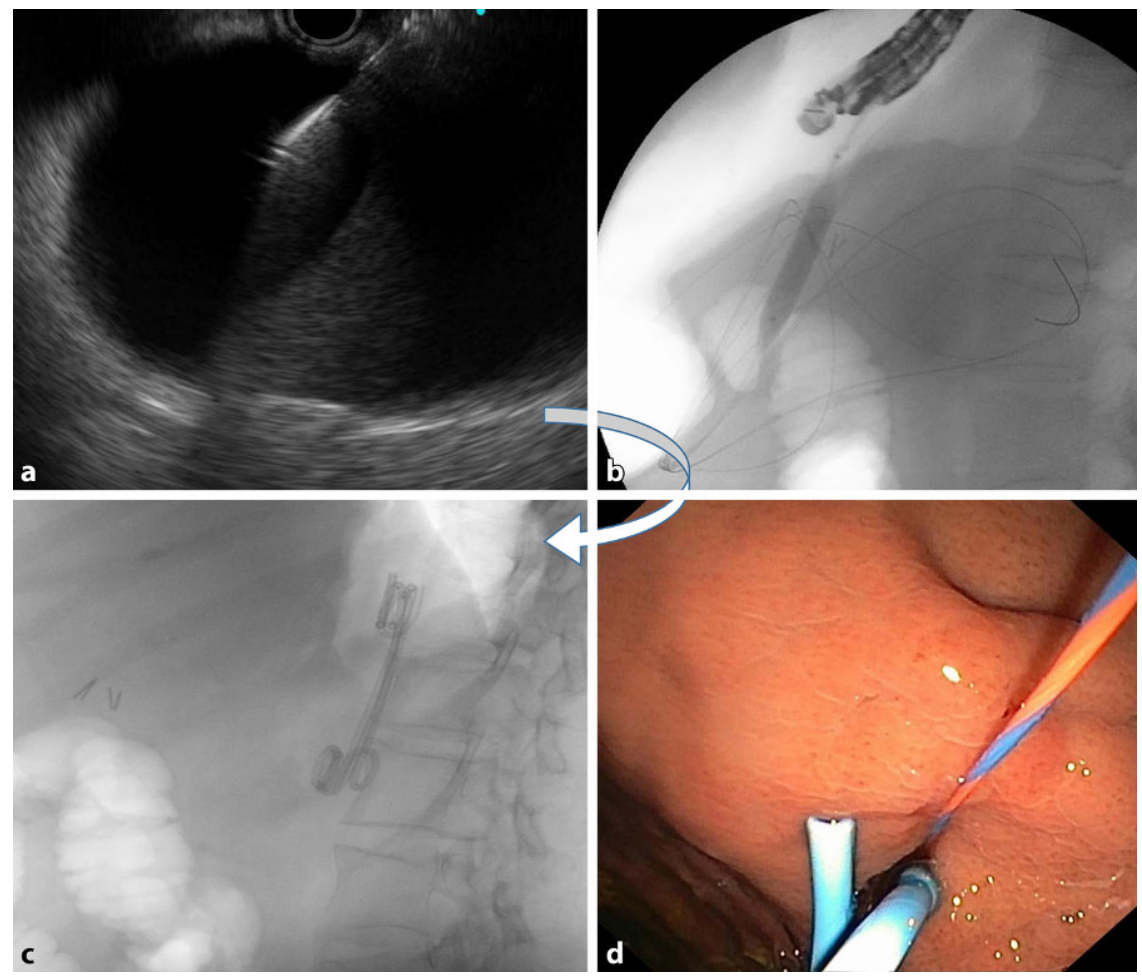

Abb. 4 \ EUS-gesteuerte Pankreaspseudozystendrainage: a EUS-gesteuerte Punktion Pseudozyste, b Ballondilatation des Zuganges, d nach Einlage des 1. Pigtails, c Endbild mit 2 Doppelpigtails. EUS Endosonographie

in das Mesenterium respektive Unterbauch. Im Fall von Superinfektionen, Schmerz- oder Kompressionssymptomen wegen Grössenzunahme ist dann individuell eine Drainage nötig. Noch vor Jahren waren diese Interventionen eine Domäne der Viszeralchirurgie. Etwa zeitgleich haben sich aber in den 1990erJahren perkutan-radiologische und endoskopisch minimal-invasive Zugänge etabliert. Beim jetzigen Wissensstand gilt der endoskopische Zugang meistens als Standardeingriff der ersten Wahl [5]. Pseudozysten und die sog. Walled-offNekrosen (WON) werden nun nach einer Planungscomputertomographie mit der EUS linear angegangen. Die Schichtbildgebung ist wichtig zur Beurteilung der Topographie und der nahe gelegenen Gefässe respektive deren Durchgängigkeit oder des Vorliegens einer Thrombose. Ist eine Kollektion weniger als $1 \mathrm{~cm}$ von der luminalen Seite des Magens oder Dünndarms ab Mukosa mit der EUSNadel erreichbar und hat eine etablierte Kapsel, kann mit einer EUS-Nadel die Kollektion punktiert und ein Draht eingebracht werden. Optische Kontrolle hierbei gibt die EUS und der gleichzeitig verwendete Bildverstärker (BV). Der mit der Nadel geschaffene Zugang wird dann mit einem Elektromesser oder Dilatationsballon eröffnet und darüber werden je nach Situation beidseits gekringelte Plastikdrains („Doppel-Pigtail“ - Abb. 4) oder voll beschichtete selbst expandierende Stents (SEMS) eingelegt. Diese SEMS sind heute meist mit beidseits breiten Tulpen ausgestattet, die die Lumina der beiden Höhlen satt komprimieren. Aus diesem Grund werden diese "lumen-apposing metal stents“ der Einfachheit halber LAMS genannt. Über diese LAMS ist ab einem inneren Durchmesser von $15-20 \mathrm{~mm}$ auch bei einer zweiten folgenden Endoskopie eine Nekrosektomie möglich. Nekrosektomien wiederum verkürzen die Hospitalisationsdauer und machen weniger Interventionen nötig. Bei Pseudozysten sind Plastikdrainagen die bevorzugten Drainagen, bei WON ist die aktuelle Datenlage noch nicht abschliessend geklärt [6]. Dies ist insofern relevant, da gewisse Stents sehr teuer sind und alle LAMS nach 3-4 Wochen durch Plastik- 
drainagen ersetzt werden sollten. Grund hierfür ist die erhöhte Blutungsgefahr durch mechanische Arrosion der LAMS in den kleiner werdenden Kollektionen.

Die Drainagen werden mindestens 3 Monate in situ belassen und im ambulanten Setting dann endoskopisch entfernt.

Mit der gleichen Technik können individuell auch pararektale Abszesse oder andere Kollektionen therapiert werden, sofern diese abgekapselt und per EUS erreichbar sind.

\section{2.b Zugang zum Gallengangs- system oder Pankreasgang}

Das Gallengangssystem der Leber und am Leberhilus wird standardmässig retrograd mithilfe einer endoskopischen retrograden Cholangiopankreatikographie (ERCP) oder aber antegrad mit einem perkutanen Zugang im Sinne einer perkutanen transhepatischen Cholangiodrainage (PTCD) erreicht. Leider gelingen diese Zugänge nicht immer. Bei ersterer Methode (ERCP) gibt es technisches Versagen bei Tumoren im Papillenbereich, oder aber der Zugang mit dem Gerät ist von Duodenum bis vor die Papille nicht möglich. Das heisst, bei Tumorinvasion und somit Magenausgangsstenose kann gar nicht bis ins Duodenum vorgespiegelt werden. Eine PTCD bringt für die Patienten eine teils störende perkutane Drainage in der rechten Flanke mit sich, die nicht selten Monate belassen werden muss und auf Zug dislozieren kann. Bei Aszites, schlanken Gallenwegen oder Lebermetastasen ist eine PTCD technisch anspruchsvoll, oder die Einlage kann frustran sein.

Als gute Alternative zu ERCP und PTCD bietet sich dann eine EUS-gesteuerte Intervention an, die je nach Topographie und Grundkrankheit nach sorgfältiger individueller Planung in verschiedener Technik vom Magen oder Duodenum her durchgeführt wird (siehe • Abb. 5).

Die sog. Hepatogastrostomie (HG, - Abb. 6) schafft EUS-gesteuert eine Verbindung von Magen in das Gallenwegssystem des linken Leberlappens mithilfe eines SEMS. Dies ist durch die anatomische Nähe und bei erweiterten Gallenwegen gut möglich. Diese Verbindung muss mit einem SEMS geschaffen werden, der peritoneal und in der Magenwand beschichtet ist. Der Stentanteil in der Leber soll unbeschichtet sein, um sich gut im Gewebe zu verankern und den Gallenabfluss aus allen Subsegmenten zu ermöglichen. Als dritte relevante Eigenschaft soll der SEMS gastral über eine breit gekragte Tulpe verfügen, um hier einen Halt zu gewährleisten. Die Galle drainiert dann gastral und es besteht bei der Schaffung der HG keine Gefahr der iatrogenen Pankreatitis wie bei einer ERCP. Die HG sind dieser Tage etabliert und können bei guter Indikation eine sehr gute Alternative der Palliation sein, ein anhaltender technischer Erfolg ist bei entsprechender Expertise in $>90 \%$ zu erwarten [7].

Extrahepatisch ist der Zugang in palliativer Situation auch in ähnlicher Art und Weise möglich, auch hier werden SEMS eingesetzt, die mithilfe der EUS platziertwerden. In benignen Fällen muss dieser Zugang als experimentell gelten.

Hier steht eine Anzeige. 


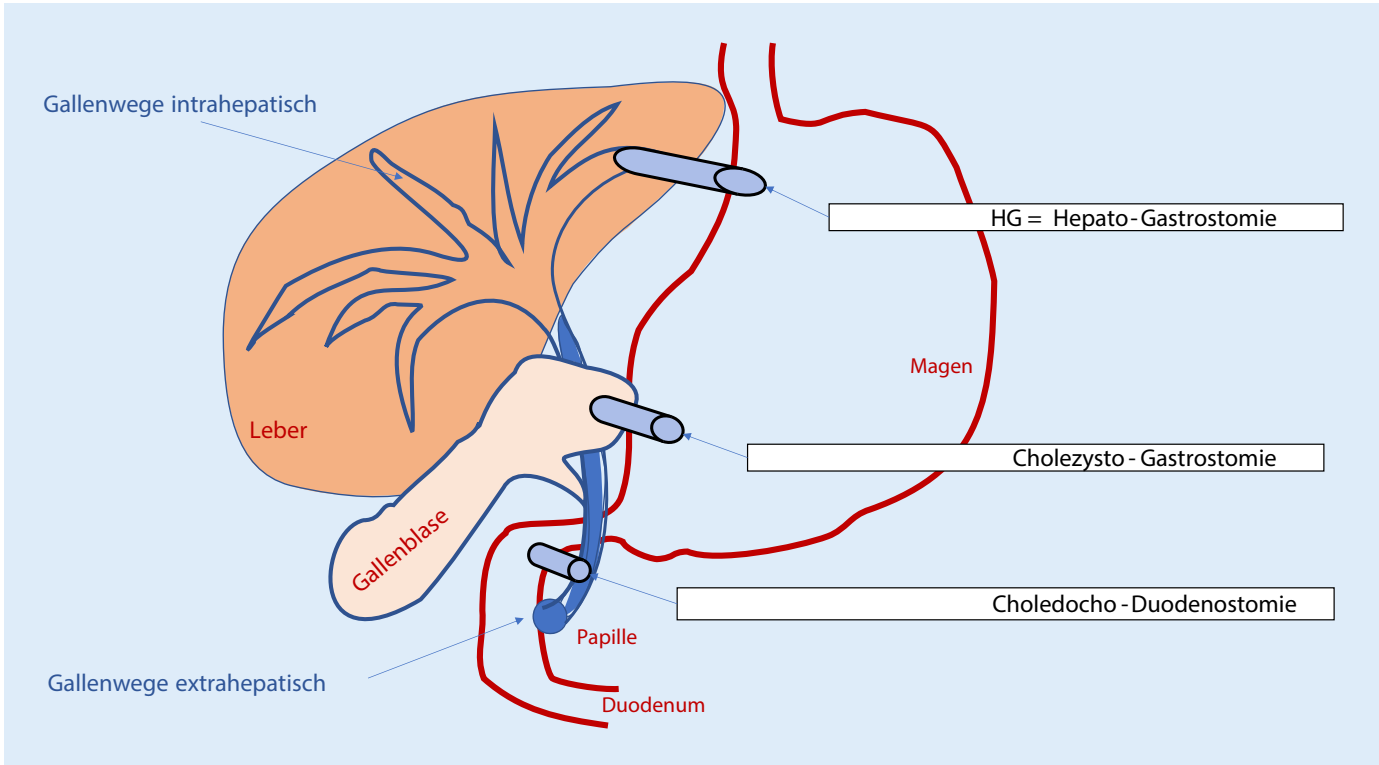

Abb. $5<$ EUS-basierte extra-anatomische Zugänge von Magen/Duodenum zu Gallenwegen \&-blase
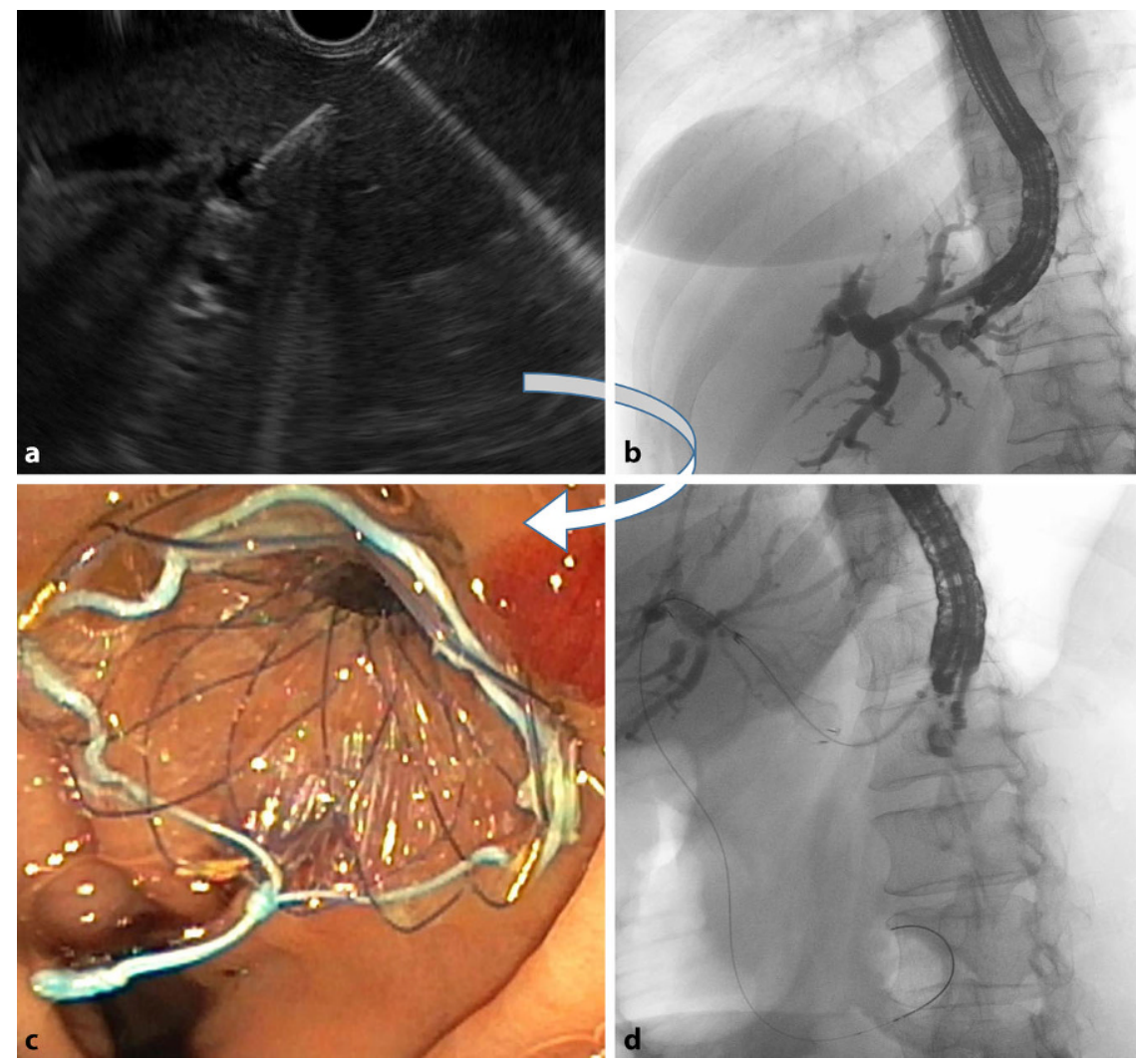

Abb. 6 \ Schaffung einer Hepatogastrostomie: a Punktion der Gallenwege des linken Leberlappens. bCholangiogramm über eineNadel.c Einbringen des Stents über einen Draht, der bis in das Duodenum liegt, $\mathbf{d}$ Stenttulpe im Magen

Mithilfe der EUS kann vom Bulbus duodeni her der Ductus hepatocholedochus gut lokalisiert und mit der Hohlnadel ein Draht eingelegt werden. Darüber geführt unter Bildverstärkerkontrolle kann mit einem kreisförmigen Elektromesser
Letztendlich ist aus dem Magenantrum EUS-gesteuert auch die Gallenblase sehr gut für eine Stenteinlage zugänglich, sodass die Galle dann in den Magen statt über die Papille abfliessen kann. Dieser Eingriff kann nötig werden bei einer akuten Cholezystitis in inoperablen $\mathrm{Pa}$ tienten, sei dies wegen Polymorbidität oder bei Kreislaufinstabilität in septischem Zustand auf der Intensivstation. Auch hier wird meistens ein LAMS verwendet, um einem sekundären $\mathrm{Ab}$ rutschen und somit einer eitrigen Peritonitis vorzubeugen. Über diesen Stent ist sogar in Einzelfällen eine Gallensteinentfernung möglich mit anschliessender endoskopischer Stententfernung. Je nach Gesamtkonstellation der Patienten ist sekundär eine Cholezystektomie doch möglich, oder der SEMS wird bei infauster Prognose belassen. Als dritte Option wird der temporäre Stent nach Abheilung der Cholezystitis endoskopisch entfernt und diese neu geschaffene Fistel verschliesst sich in den meisten Fällen spontan innert Tagen.

Der Pankreasgang wiederum kann auch sehr gut vom Magen her endosonographisch lokalisiert und punktiert werden. Ist bei einer ERCP der Pankreasgang von der Papille her nicht sondierbar wegen einer Tumorkompression oder entzündlichen Stenose, kann ein antegrades Angehen zur Dekompression sinnvoll sein. Bis dato war diese Druckreduktion des Gangs nur chirur- 


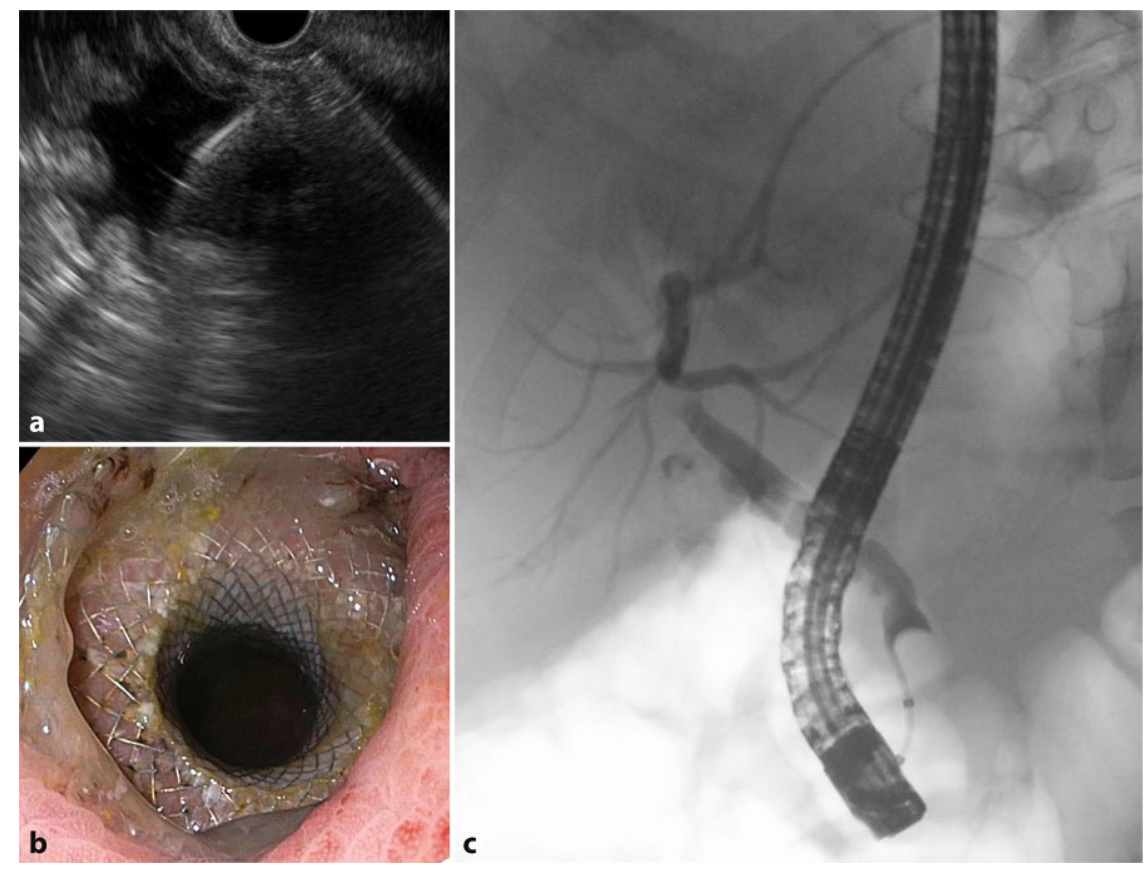

Abb. $7 \Delta$ Zugang in den nativen Magen nach Magenbypassoperation mithilfe eines LAMS. a EUS-geführte Punktion des nativen Magen und Füllen mit Flüssigkeit, b Sicht auf ein gebrachen LAMS zu Restmagen, c Duodenoskop durch LAMS mit Cholangiogramm bei ERCP. LAMS "lumen-apposing metal stent", EUS Endosonographie, ERCP endoskopische retrograde Cholangiopankreatikographie

gisch möglich; sei dies durch Resektion des betroffenen Pankreasanteiles, oder aber durch eine eröffnende Technik mit z. B. Aufnähen einer Dünndarmschlinge. Durch den EUS-gesteuerten Zugang mit Punktion eines erweiterten Pankreasgangs mit Einlage einer Plastikdrainage in den Magen ist nun erstmals ein minimal-invasiver Zugang möglich. Im besten Fall gelingt es, einen Draht transpapillar zu platzieren, sodass dann mit einer zusätzlichen ERCP im Sinne eines Rendez-vous-Verfahrens die Stenose mit einer Drainage oder selten einem Stent überbrückt werden kann.

\section{Schaffung einer Verbindung im Gastrointestinaltrakt}

Im letzten Jahrzehnt wurden zuverlässige Daten betreffend neu kreierter Verbindungen von Magen oder Duodenum zu Gallenwegen/-blase oder retroperitonealen Kollektionen erhoben. Diese formal iatrogenen Perforationen zweier Hohlorgane mit temporärer Passage des Peritoneums werden mit tiefem Komplikationsrisiko durchgeführt und verhindern oft grössere chirurgische Eingriffe.
Als neueste Errungenschaft dieser interventionellen EUS dürfen die Zugänge in den nativen Magen nach bariatrischer Chirurgie und die Schaffung der endoskopischen Gastroenterostomie beschrieben werden.

Bei ersterem Eingriff wird bei Patienten nach Magenbypass der Zugang zum nativen Magen mithilfe eines voll beschichteten Stents im Sinne eines LAMS wieder geschaffen. Wichtigste Indikation kann die Notwendigkeit einer ERCP sein, wenn mit den aufwändigeren Endoskopien (Single- oder Doppelballonenteroskopien) der Magen retrograd nicht erreicht werden kann. Mithilfe der EUS wird der Magen identifiziert und mit einer Nadel punktiert. Darüber kann dieser mit über einem Liter Flüssigkeit gefüllt werden und wird somit vom Magenpouch her gut sichtbar in der EUS. Im 2. Schritt kann dann erneut mithilfe der EUS ein Stent in den Magen eingebracht werden, indem vorgängig mit einem ringförmigen Messer der Zugang "geschnitten“ wird (- Abb. 7). Wenige Tage nachher kann dieser Magenzugang für eine ERCP in üblicher Weise verwendet werden. Die technische Erfolgsrate ist mit dieser Methode mit gegen $100 \%$ vs. $60 \%$ mithil- fe von ballonassistierten Enteroskopien deutlich höher bei gleicher Komplikationsrate von 10 respektive $7 \%$ [8].

Bei Magenausgangsstenosen in $\mathrm{Tu}$ morpatienten wird die stark störende Outlet-Symptomatikentweder mit Einlage eines unbeschichteten Duodenalstents oder mit der chirurgischen Schaffung einer Gastroenterostomie (GE) angegangen. Ersterer bleibt oft nicht länger als 6 Monaten durchgängig und die chirurgische GE bedarf einer Narkose und eines viszeralchirurgischen Eingriffs bei oftmals schwer kranken Patienten.

Auch hier kann die interventionelle EUS eine gut funktionierende Alternative anbieten. Nach Gabe von Spasmolytika und luminaler Füllung des freien Jejunums durch eine tief eingelegte Sonde, kann mit der EUS oft eine Dünndarmschlinge vom Magen her gut visualisiert werden. Bei kurzem Abstand ist es wiederum mit gleicher EUS-Technik möglich, eine Fistel zwischen Magen und Jejunum zu schaffen. Hier wird in einem Arbeitsgang mit einem Elektromesser Magen und Jejunum axial eröffnet und darüber das Stenteinführbesteck eingeschoben. Der Eingriff tangiert somit vorübergehend das freie Peritoneum und es muss zwingend ein LAMS zum Einsatz kommen. Bei Entfalten hält dann je eine Tulpe das Lumen im Jejunum und im Magen und bringt beide Lumina dicht aneinander, damit keine Peritonitis auftreten kann (• Abb. 8). Der technische und klinische Erfolg ist trotz kleinen $\mathrm{Pa}$ tientenzahlen überzeugend [9], der Eingriff selbst zeitlich kurz und in Sedation durchzuführen. Nach unserer Erfahrung sind die Patienten bereits einen Tag postinterventionell in der Lage, flüssige Nahrung zu sich zu nehmen. Prospektive Daten im Vergleich zur chirurgischen GE müssen die vermutete Überlegenheit im palliativen Setting noch erbringen. Medizinisch-theoretisch kann die endoskopische GE auch bei benignen Erkrankungen zum Einsatz kommen.

Zusammengefasst hat sich die EUS in der Diagnostik selbst weiterentwickelt und kann mit Kontrastmittel, Elastographie und sogar Realtime-Mikroskopie aufwarten. Zusätzlich ist das therapeutische Armamentarium enorm gewachsen: Nebst Injektionen zur Blutstillung oder 

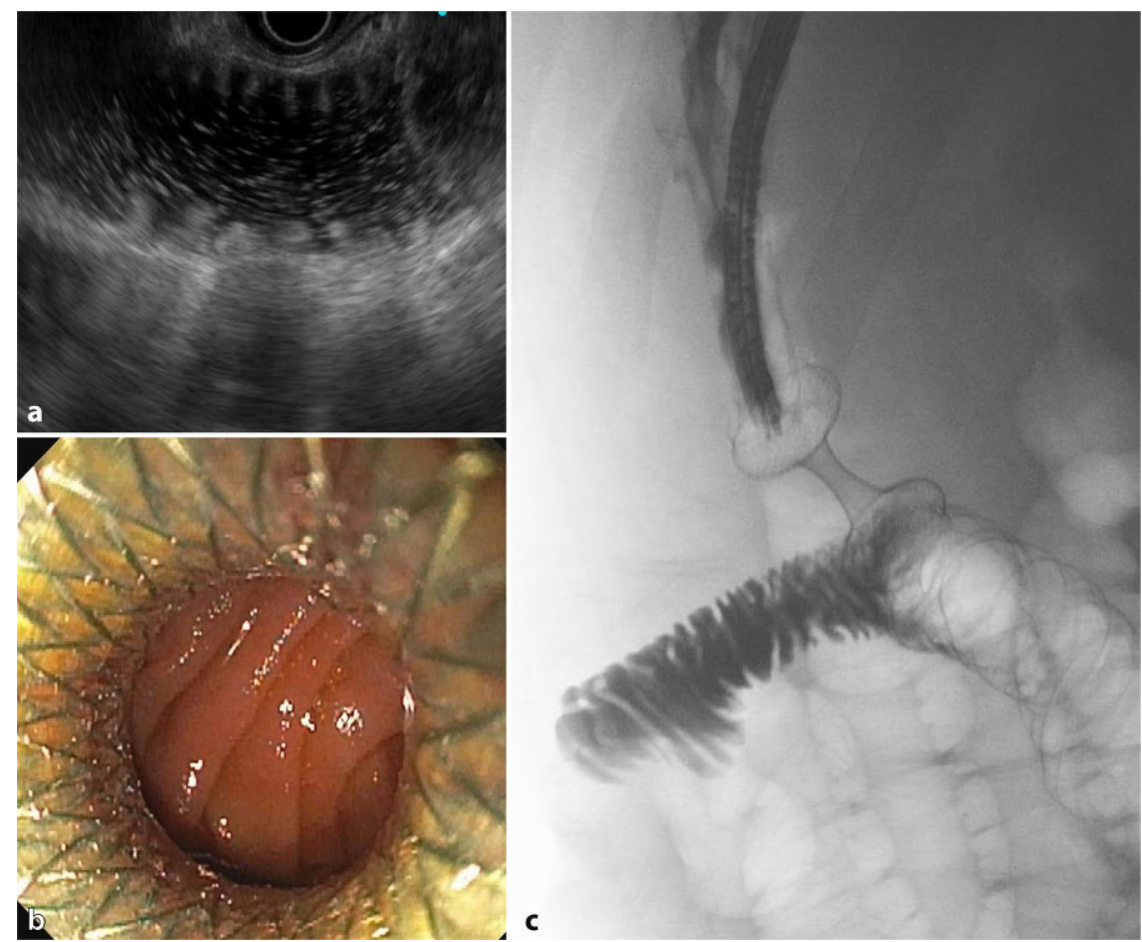

Abb. $8 \Delta$ Schaffung einer Gastroenterostomie mithilfe eines LAMS. a Darstellung einer dilatierten Jejunumschlinge hinter dem Magen, $\mathbf{b}$ Eingebrachter LAMS mit Blick auf Jejunum-Lumen, $\mathbf{c}$ Kontrastmitteldarstellung der Verbindung Magen-Jejunum. LAMS "lumen-apposing metal stent"

Analgesie können pankreatitisassoziierte Kollektionen intern suffizient drainiert werden und machen oft chirurgische Eingriffe unnötig. Die EUS-gesteuerten Gallengangsdrainagen wiederum sind gute Alternativen zur ERCP und PTCD. Letztendlich können auch minimalinvasiv Verbindungen im Gastrointestinaltrakt geschaffen werden, sei dies als Zugang zum Magen nach Bypassoperation oder Dünndarm bei der Schaffung einer endoskopischen Gastroenterostomie.

\section{Take-home-Message}

\section{- Die therapeutische Endosonogra-} phie, interventionelle EUS genannt, ist im klinischen Alltag angekommen.

- EUS-gesteuerte Gewebeentnahmen, Magenvarizentherapien, Drainagen von Kollektionen, wie Pseudozysten und Walled-off-Nekrosen nach Pankreatitis, und Drainagen von perirektalen/retroperitonealen Abszessen sind etabliert.

- Intra- und extrahepatische Gallenwegsdrainagen sind gute neue Alternativen zur ERCP und PTCD.

\section{- Die EUS-unterstützte Schaffung eines Zugangs zum Magen oder Dünndarm stellen attraktive Alternativen zu chirurgischen Eingriffen dar.}

\section{Korrespondenzadresse}

\section{Prof. Dr.med. C. Gubler}

Klinik für Gastroenterologie und Hepatologie, UniversitätsSpital Zürich

Rämistrasse 100, 8091 Zürich, Schweiz

christoph.gubler@usz.ch

Funding. Open access funding provided by University of Zurich

\section{Einhaltung ethischer Richtlinien}

Interessenkonflikt. B. Morell, F.O. The und C. Gubler geben an, dass kein Interessenkonflikt besteht.

Für diesen Beitrag wurden von den Autoren keine Studien an Menschen oder Tieren durchgeführt. Für die aufgeführten Studien gelten die jeweils dort angegebenen ethischen Richtlinien.

Open Access Dieser Artikel wird unter der Creative Commons Namensnennung 4.0 International Lizenz veröffentlicht, welche die Nutzung, Vervielfältigung Bearbeitung, Verbreitung und Wiedergabe in jeglichem Medium und Format erlaubt, sofern Sie den/die ursprünglichen Autor(en) und die Quelle ordnungsgemäß nennen, einen Link zur Creative Commons Lizenz beifügen und angeben, ob Änderungen vorgenommen wurden.

Die in diesem Artikel enthaltenen Bilder und sonstiges Drittmaterial unterliegen ebenfalls der genannten Creative Commons Lizenz, sofern sich aus der Abbildungslegende nichts anderes ergibt. Sofern das betreffende Material nicht unter der genannten Creative Commons Lizenz steht und die betreffende Handlung nicht nach gesetzlichen Vorschriften erlaubt ist, ist für die oben aufgeführten Weiterverwendungen des Materials die Einwilligung des jeweiligen Rechteinhabers einzuholen.

Weitere Details zur Lizenz entnehmen Sie bitte der Lizenzinformation auf http://creativecommons.org/ licenses/by/4.0/deed.de.

\section{Literatur}

1. Napoleon B, Palazzo M, Lemaistre Al, Caillol $F_{\text {, }}$ Palazzo L, Aubert A, Buscail L, Maire F, Morellon BM, Pujol B, Giovannini M (2019) Needle-based confocal laser endomicroscopy of pancreatic cystic lesions: a prospective multicenter validation study in patients with definite diagnosis. Endoscopy 51(9):825-835

2. GressF, SchmittC, Sherman S, CiacciaD, IkenberryS, Lehman G (2001) Endoscopic ultrasound-guided celiac plexus block for managing abdominal pain associated with chronic pancreatitis: a prospective single center experience. Am J Gastroenterol 96(2):409-416

3. Gubler C, Bauerfeind P (2014) Safe and successful endoscopic initial treatment and longterm eradication of gastric varices by endoscopic ultrasound-guided Histoacryl (N-butyl-2cyanoacrylate) injection. Scand J Gastroenterol 49(9):1136-1142

4. Barthet M, Giovannini M, Lesavre N, Boustiere $C$ Napoleon B, Koch S, Gasmi M, Vanbiervliet G, Gonzalez J-M (2019) Endoscopic ultrasoundguided radiofrequency ablation for pancreatic neuroendocrine tumors and pancreatic cystic neoplasms: a prospective multicenter study. Endoscopy 51(9):836-842

5. Khan MA, Kahaleh M, Khan Z, Tyberg A, Solanki S, Haq KF, Sofi A, Lee WM, Ismail MK, Tombazzi C, Baron TH (2019) Time for a changing of guard: from minimally invasive surgery to endoscopic drainage for management of pancreatic walled-off necrosis. JClin Gastroenterol 53(2):81-88

6. Hammad T, Khan MA, Alastal Y, Lee W, Nawras A Kashif Ismail M, Kahaleh M (2018) Efficacy and Safety of Lumen-Apposing Metal Stents in Management of Pancreatic Fluid Collections: Are They Better Than Plastic Stents? A Systematic Review and Meta-Analysis. Dig Dis Sci 63(2):289-301

7. Poincloux L, Rouquette O, Buc E, Privat J, Pezet $D$, Dapoigny M, Bommelaer G, Abergel A (2015) Endoscopic ultrasound-guided biliary drainage after failed ERCP: cumulative experience of 101 procedures at a single center. Endoscopy 47(9):794-801

8. Bukhari M, Kowalski T, Nieto J, Kunda R, Ahuja NK, Irani S, Shah A, Loren D, Brewer O, Sanaei O, Chen YI, Ngamruengphong $S$, Kumbhari V, Singh V, Dakour Aridi H, Khashab MA (2018) An international, multicenter, comparative trial 
of EUS-guided gastrogastrostomy-assisted ERCP versus enteroscopy-assisted ERCP in patients with Roux-en-Y gastric bypass anatomy. Gastrointest Endosc 88(3):486-494

9. Khashab MA, Bukhari M, Baron TH, Nieto J, El Zein M, Chen YI, Chavez YH, Ngamruengphong S, Alawad AS, Kumbhari V, Itoi T (2017) International multicenter comparative trial of endoscopic ultrasonography-guided gastroenterostomy versus surgical gastrojejunostomy for the treatment of malignant gastric outlet obstruction. Endosc Int Open 5(4):E275-E281

Hinweis des Verlags. Der Verlag bleibt in Hinblick auf geografische Zuordnungen und Gebietsbezeichnungen in veröffentlichten Karten und Institutsadressen neutral.

\section{S.F. Schoppmann, M. Riegler Multidisciplinary Management of Gastroesophageal Reflux Disease}

Das multidisziplinäre Management der Gastroösophagealen Refluxkrankheit

Springer International Publishing 2021, 1. Auflage, 217 S., 19 s/w Abbildungen, 66 farbige Abbildungen Abb., (ISBN: 978-3-030-53750-0), Hardcover 145,59 EUR

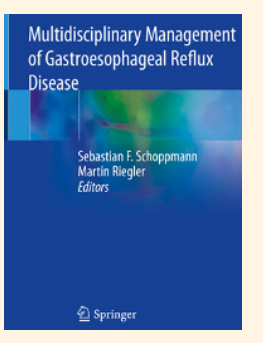

Die gastroösophageale Refluxkrankheit (GERD) zählt heute zu den häufigsten Lebensstilerkrankungen. Diagnose und Behandlung dieser Erkrankung stellen eine beträchtliche Herausforderung dar und erfordern einen multidisziplinären Ansatz. Das vorliegende Werk wurde von zwei hervorragenden GERD-Experten herausgegeben, Sebastian F. Schoppmann und Martin Riegler, gemeinsam mit weiteren herausragenden, weltweit anerkannten Koautoren. In diesem Buch wird ein klarer und umfassender Überblick zum gegenwärtigen Stand des multidisziplinären GERD-Managements geboten. Es werden alle Aspekte der Diagnose und des Managements der GERD, inklusive Endoskopie, Gastroenterologie, Radiologie, Pulmologie, Chirurgie, Palliation und interventionellen Medizin, auf hohem Niveau präsentiert und diskutiert. Darüber hinaus werden anatomische, physiologische, histopathologische und pathophysiologische Charakteristika des Ösophagus aufgezeigt. Multi- und interdisziplinäre Ansätze zur GERD und zum Barrett-Ösophagus (BE) einschließlich der neuesten Erkenntnisse im Verständnis dieser Erkrankungen, wie auch Überlegungen zu rezenten diagnostischen und therapeutischen Verfahren werden abgedeckt. Die fundierte Expertise der Autoren und hochpräzise Literaturrecherche erlaubt die Darstellung aller derzeit bekannten Aspekte der GERD und des BE auf hohem Niveau. Insbesondere wird durch die Präsentation neuester Methoden in der Therapie das breite Spektrum des heute zur Verfügung stehenden Behandlungsinstrumentariums demonstriert und damit das Tor zu einer individualisierten Therapie geöffnet.

In Abschnitt 1 wird die Pathophysiologie von Verletzungen des unteren Ösophagussphinkters dargestellt und ein neues pathologisches Testverfahren für derartige Verletzungen präsentiert. Abschnitt 2 umfasst detaillierte Informationen zur ösophagealen Funktionstestung auf GERD. Die Abschnitte 3 und 4 behandeln die Endoskopie und endoskopische ablative Therapien bei GERD und BE. Es werden die endoskopische Mukosaresektion, die endoskopische Submukosadissektion, die Radiofrequenzablation sowie die Kryoballonablation beschrieben. Die dazugehörigen Diagramme und Abbildungen helfen, die diversen Verfahren auch für den Nichtspezialisten verständlich zu machen. Aktuelle Studien zum endoskopischen Management der GERD und des BE werden besprochen. In Abschnitt 5 wird auf die Bedeutung der Bildgebung für die Diagnose und Darstellung zusätzlicher Pathologien vor Durchführung einer Antirefluxchirurgie eingegangen - sowie auf ihren Wert in der Diagnose von Früh- und Spätkomplikationen. Abschnitt 6 ist den diversen extraösophagealen Manifestationen der GERD gewidmet, u.a. dem chronischen Husten, dem Globussyndrom, der Heiserkeit, dem Stimmlippengranulom und der Stimmlippendysfunktion und der chronischen Rhinosinusitis. Chirurgisch orientierte Leser werden über die Abschnitte 7 bis 10 erfreut sein. Begleitet von eindrucksvollen Abbildungen werden im Text die verschiedenen Verfahren der Fundoplicatio vorgestellt. Insbesondere wird im achten Abschnitt eines der neuartigen Instrumente in der GERD-Therapie präsentiert. Hier wird auf die Technik und Evidenz der magnetischen Sphinkteraugmentation eingegangen. Abschnitt 11 ist der Diagnose des Barrett-Karzinoms unter Einsatz diverser bildgebender Techniken gewidmet. Zu guter Letzt gibt es zwei Kapitel zum ösophagealen Adenokarzinom, einschließlich der chirurgischen Behandlung und Palliation. Die ausgezeichnete Editierung wird durch aktuelle Literaturübersichten, Tabellen, hochqualitative Abbildungen und ein Stichwortverzeichnis bereichert.

Zusammenfassend präsentiert dieses Werk alle Aspekte des multidisziplinären Managements der gastroösophagealen Refluxerkrankung, einschließlich neuartiger diagnostischer und therapeutischer Konzepte und Strategien. Dieses Buch ist eine wertvolle Ergänzung für die Bibliothek jedes Mediziners, der sich mit diesem Thema beschäftigt. 